\title{
Tracheal cancer treated with a short course of external and endoluminal radio-chemotherapy combined with cetuximab - a case report
}

\author{
Aikaterini Papadopoulou, Marios Froudarakis, loannis Abatzoglou, Michael I. Koukourakis \\ Departments of Radiotherapy/Oncology and Pneumonology, Democritus University of Thrace, Alexandroupolis, Greece
}

\begin{abstract}
Primary tumors of the trachea are rare. Such cases are presented with acute respiratory distress demanding immediate therapeutic intervention. Herein, we present a case of an unresectable second primary tracheal cancer treated with intraluminal brachytherapy ( $8 \mathrm{~Gy}$ at $1 \mathrm{~cm}$ from catheter) followed by a short course of external beam hypofractionated radiotherapy $(4.5 \mathrm{~Gy} \times 4$ fractions) and a final brachytherapy fraction $(8 \mathrm{~Gy})$, delivering a biological dose higher than $57.5 \mathrm{~Gy}$ (for $\alpha / \beta=4 \mathrm{~Gy}$ ) to the tumor within 4 weeks. Concurrent chemotherapy consisted of: fluoruracil $\left(1000 \mathrm{mg} / \mathrm{m}^{2}\right)$, leucovorin $\left(100 \mathrm{mg} / \mathrm{m}^{2}\right)$, oxaliplatin $\left(80 \mathrm{mg} / \mathrm{m}^{2}\right)$ and cetuximab $\left(500 \mathrm{mg} / \mathrm{m}^{2}\right)$, administered every two weeks for two consecutive cycles. Complete response was evident during the second brachytherapy fraction and the patient is alive with no evidence of disease, two years after therapy, without any late radiation sequel.
\end{abstract}

Key words: brachytherapy, cetuximab, radiotherapy, trachea cancer.

\section{Purpose}

Primary tumors of the trachea are rare and accounting for $0.1 \%$ of all malignancies [1-3]. The experience in the management of primary tracheal tumors remains limited because of rarity of the disease. Some centers suggest surgical resection as the treatment of choice (tracheal, laryngotracheal or carinal resection) followed by radiotherapy alone or combined with chemotherapy, although the results are uncertain [4]. However, national epidemiologic studies of tracheal carcinoma have demonstrated disappointingly poor long term survival $(<15 \%)$ and exceedingly low rates of surgical resection $(<10 \%)$ [1-3]. Usually, tracheal malignancies are diagnosed too late for curative resection. Radiotherapy, either alone or combined with chemotherapy and/or endotracheal desobstruction, is the primary treatment modality for unresectable tumors [5]. Due to its accessibility, the trachea is a reasonable target for external beam combination with intraluminal radiotherapy [6]. Herein, we present a case of unresectable second primary tracheal cancer treated with such therapeutic approach combined with concurrent chemotherapy.

\section{Case presentation}

A 56-year-old male patient was admitted to our department for postoperative RT of the larynx. In the past year, he had had a chordectomy for a squamous cell laryngeal cancer, staged as $\mathrm{T} 2 \mathrm{~N} 0$ without any postoperative treatment. After second laser incomplete resection, due to local relapse of the disease, he received radiotherapy confined to the larynx (LINAC - 6MV - 20 fractions -5 fractions per week - 2.7 Gy per day - normalized total dose calculated for $\alpha / \beta=4$ Gy: $60.3 \mathrm{~Gy}$ ) in combination with cisplatin (30 mg/ $\mathrm{m}^{2}$ per week). At the time, fiberoptic bronchoscopy of tracheobronchial tree was negative for endoluminal lesion. Two years later, he developed second primary tracheal cancer, while the patient was free from laryngeal disease. He was admitted for acute respiratory distress associating dyspnea, wheezing, cough and hemoptysis. Bronchoscopy revealed a tumor localized at the middle/lower part of the trachea causing $80 \%$ of the lumen obstruction. Histology revealed a squamous cell carcinoma. The patient was immediately supported with high dose dexamethasone (8 $\mathrm{mg}$ thrice a day i.v.) and, after the placement of an intratracheal catheter through the fiberoptic bronchoscope under local anesthesia, brachytherapy was performed using the Microselectron Ir192-HDR (Nucletron ${ }^{\circledR}$ ). A dose of 8 Gy was delivered, calculated at $1 \mathrm{~cm}$ from the catheter (Fig. 1A), 95\% of isodose curves extending $2 \mathrm{~cm}$ above and below the radiologically detectable disease. One week later, external beam RT was applied by delivering 4 consecutive fractions of $4.5 \mathrm{~Gy}$, using 6/18 MV photons and three-field conformal technique (Fig. 1B). The target volume included the trachea and paratracheal lymph nodes. Intravenous chemothera- 
py consisted of: fluoruracil $\left(1000 \mathrm{mg} / \mathrm{m}^{2}\right)$, leucovorin $\left(100 \mathrm{mg} / \mathrm{m}^{2}\right)$, oxaliplatin $\left(80 \mathrm{mg} / \mathrm{m}^{2}\right)$ and cetuximab $\left(500 \mathrm{mg} / \mathrm{m}^{2}\right)$. Despite the complete regression of the tumor (Fig. 1C), the second fraction of brachytherapy (8 Gy) was delivered one month after the first brachytherapy fraction to secure eradication of the disease. This regimen delivered normalised total dose equivalent higher than $57.5 \mathrm{~Gy}$ (for $\alpha / \beta=4$ Gy) to the tumor within 4 weeks. The only complication reported during therapy was grade 1 dysphagia. Two years after the treatment, the patient has no clinical or radiological evidence of the disease, nor late complications from the treatment.

\section{Discussion}

The delay in diagnosis due to non-specific symptoms at the early stage of the tracheal tumor growth results in a sharp onset of symptomatology, demanding urgent treatment in order to avoid complete obstruction of the airways. However, laser therapy is very useful in improving the obstructive symptoms, RT is the only curative method resulting in a long term control of the disease [7]. External beam doses higher than 60 Gy appear to provide good median survival time and local control, although are associated with increased complications (tracheoesophageal fistula, esophageal stricture, and severe tracheal crusting) [7].

Endobronchial high-dose-rate brachytherapy has been used for tracheal carcinomas as a boost to external beam irradiation. Total dose and dose per fraction have not been well recognized, up to the present moment. Reports on the combination of external and endoluminal radiotherapy are rare. Due to the infrequency of the disease the role of endoluminal brachytherapy in the treatment regiment of tracheal neoplasms has not been established. In a study by Harms et al., in 10 cases treated with external beam radiotherapy (60 Gy) followed by a booster endoluminal brachytherapy dose, the median survival was 33 months [6]. The efficacy of combined radio-chemotherapy is also uncertain. The new current approach in oncology is the use of molecular targeted therapies. Cetuximab, an anti-EGFR monoclonal antibody, is a targeted therapy approved for the treatment of locally advanced squamous cell carcinoma of the head and neck, in association with radiotherapy, following a randomized trial confirming doubling of the median survival [8]. The EGFR (epidermal growth factor receptor) is a type 1 trans-membrane receptor tyrosine kinase that is over expressed in epithelial tumors. EGFR expression is associated with inferior survival, radioresistance, and loco-regional failure. It is observed that the radiation increases the expression of EGFR in cancer cells and the blockade of EGFR sensitizes the tumor cells to the effects of radiation. We previously showed that cetuximab can be combined safely with platinum and radiotherapy [9]. In addition, there are more recent data showing good survival benefit for patients with recurrent/ metastatic disease who were treated with 1 st-line regiment of platinum, fluorouracil and cetuximab. These promising results have had a significant impact on the standard of care for squamous carcinomas [10].
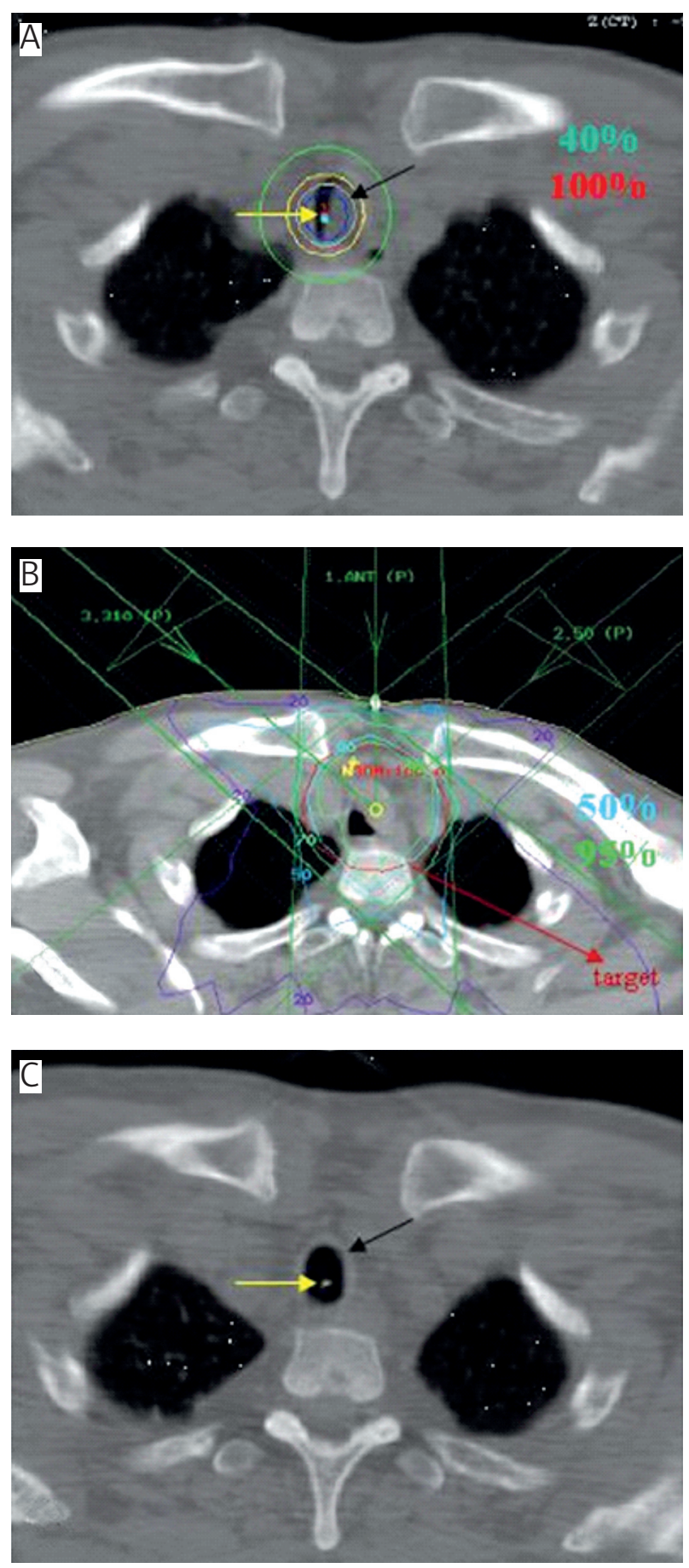

Fig. 1. A tracheal tumor obstructing the trachea (black arrow); (A) isodose curves around the intratracheal cactheter (yellow arrow); (B) three-field conformal planning for external beam hypofractionated radiotherapy; (C) complete remission of the tumor (black arrow) during the $2^{\text {nd }}$ endoluminal brachytherapy, one month later

\section{Conclusions}

The evaluation of the fractionation, total dose and the optimal chemotherapy regimen for tracheal carcinomas are difficult to acquire from future trials due to infrequency of 
the disease. The presented case is significant due to rapid complete response, long term control and the lack of radiation sequel achieved with a short schedule of external and endoluminal radiotherapy in combination with chemotherapy and cetuximab.

\section{References}

1. Manninen MP, Antila PJ, Pukander JS et al. Occurrence of tracheal carcinoma in Finland. Acta Otolaryngol 1991; 111: 1162 1169.

2. Licht PB, Friis S, Pettersson G. Tracheal cancer in Denmark: a nationwide study. Eur J Cardiothorac Surg 2001; 19: 339-345.

3. Honings J, van Dijck JA, Verhagen AF et al. Incidence and treatment of tracheal cancer: a nationwide study in the Netherlands. Ann Surg Oncol 2007; 14: 968-976.

4. Yamamoto K, Kosaba S, Ikeda T. Tumors involving the tracheal carina: new technique of carinal reconstruction. Ann Thorac Surg 2000; 70: 1419-1422.

5. Compeau CG, Keshavjee S. Managment of tracheal neoplasms. Oncologist 1996; 1: 347-353.

6. Harms W, Latz D, Becker H et al. Treatment of primary tracheal carcinoma. The role of external and endoluminal radiotherapy. Strahlenther Onkol 2000; 176: 22-27.

7. Chow DC, Komaki R, Libshitz HI et al. Treatment of primary neoplasms of the trachea. The role of radiation therapy. Cancer 1993; 71: 2946-2952.

8. Bonner JA, Harari PM, Giralt J et al. Radiotherapy plus cetuximab for squamous-cell carcinoma of the head and neck. N Engl J Med 2006; 354: 567-578.

9. Koukourakis MI, Tsoutsou PG, Karpouzis A et al. Radiochemotherapy with cetuximab, cisplatin, and amifostine for locally advanced head and neck cancer: a feasibility study. Int J Radiat Oncol Biol Phys 2010; 77: 9-15.

10. Vermorken JB, Mesia R, Rivera F et al. Platinum-based chemotherapy plus cetuximab in head and neck cancer. N Engl J Med 2008; 359: 1116-1127. 